\title{
Characteristics of Ni-Doped IZO Layers Grown on IZO Anode for Enhancing Hole Injection in OLEDs
}

\author{
Kwang-Hyuk Choi, ${ }^{a}$ Jin-A Jeong, ${ }^{a}$ Han-Ki Kim, ${ }^{\text {a, }}$ Jae-Young Lee, \\ Jung-Hwan Lee, ${ }^{b}$ Hyo-Dae Bae, ${ }^{\text {b }}$ Yoon-Heong Tak, ${ }^{\text {b }}$ Se-Hyung Lee, \\ Dong-Seok Leem, ${ }^{\mathrm{c}}$ and Jang-Joo Kim ${ }^{\mathrm{c}}$
}

${ }^{a}$ School of Advanced Materials and Systems Engineering, Kumoh National Institute of Technology, Gumi 730-701, South Korea

${ }^{b}$ LG Display, OLED Panel Development Team, Jinpyung-dong, Gumi 730-726, South Korea

${ }^{c}$ School of Materials Science and Engineering, Seoul National University and Organic Light Emitting

Diodes Center, Sillim-dong, Seoul 151-741, South Korea

\begin{abstract}
The preparation and characteristics of a Ni-doped indium zinc oxide (NIZO) layer were investigated to enhance hole injection in organic light emitting diodes (OLEDs). A thin NIZO layer with a thickness of $5 \mathrm{~nm}$ was cosputtered onto an indium zinc oxide (IZO) anode using tilted $\mathrm{Ni}$ and IZO dual targets de magnetron sputtering at room temperature in a pure Ar atmosphere. Using $3 \mathrm{~W}$ of Ni dc power, we can obtain a NIZO $(5 \mathrm{~nm}) / \mathrm{IZO}(135 \mathrm{~nm})$ double-layer anode with a sheet resistance of $30.04 \Omega / \square$ and an optical transmittance of $83.8 \%$ at a wavelength of $550 \mathrm{~nm}$. In addition, it was found that the work function of the NIZO layer was higher than that of a pure IZO anode due to the presence of a $\mathrm{NiO}_{x}$ phase in the NIZO layer. An increase of Ni dc power above $7 \mathrm{~W}$ significantly degrades the electrical and optical properties in the NIZO layer. X-ray diffraction examination demonstrated that the NIZO layer consisted of an amorphous structure regardless of the $\mathrm{Ni}$ dc deposition power due to low substrate temperature. Furthermore, an OLED fabricated on the NIZO layer exhibited a higher current density, luminance, and efficiency due to improved hole injection by the high work function NIZO. These results indicate that the NIZO/IZO anode scheme is a promising anode material system for enhancing hole injection from the anode into the active layer of OLEDs. (c) 2008 The Electrochemical Society. [DOI: 10.1149/1.2979143] All rights reserved.
\end{abstract}

Manuscript submitted June 19, 2008; revised manuscript received August 7, 2008. Published September 25, 2008.

Indium tin oxide (ITO) films deposited on glass substrate have been academically and industrially used as transparent electrodes for organic light emitting diodes (OLEDs) and flexible OLEDs due to their visible transparency $(\sim 90 \%)$ and low resistivity (2-4 $\left.\times 10^{-4} \Omega \mathrm{cm}\right){ }^{1-5}$ High-performance OLEDs and flexible OLEDs require a high-quality ITO anode layer with low resistance, high transparency, chemical stability, and high work function because hole injection and light-emitting properties are critically impacted by the ITO anode layer. ${ }^{6}$ Unfortunately, conventional ITO anodes have several drawbacks, such as an imperfect work function alignment with organic materials, chemical instability, high process temperature, and easy deterioration of the ITO target. In particular, the high energy barrier between ITO and the highest occupied molecular orbit of organic materials prevents effective hole injection from the ITO into the organic layer. For these reasons, several approaches have been employed to increase the work function of ITO, including wet treatment, ${ }^{7,8}$ plasma treatment, ${ }^{9,10} \mathrm{UV}$ ozone treatment, ${ }^{11}$ selfassembly monolayer coating treatment, ${ }^{12}$ and Ni doping of ITO. ${ }^{13,14}$ In particular, several groups have reported that $\mathrm{Ni}$ cosputtering with ITO or Ni doping on the surface region of an ITO can potentially enhance hole injection into OLEDs due to the effect of the p-type transparent conductive $\mathrm{NiO}_{x}$ layer. ${ }^{13-15}$ Because the $\mathrm{NiO}_{x}$ layer is a well-known p-type conductor with high work function, it is more desirable for hole injection into the organic layer than ITO. ${ }^{16}$ Similar to ITO anode films, the investigation of $\mathrm{Ni}$ doping or cosputtering with indium zinc oxide (IZO) is therefore necessary to improve the performance of IZO anodes. However, the characteristics and application of Ni-doped IZO (NIZO) anodes have not been investigated in detail, even though the electrical and optical properties of IZO anode are well-known. ${ }^{17}$

In this work, we investigated the electrical, optical, structural, and surface properties of a NIZO layer grown by dual-target dc sputtering at room temperature. Despite the NIZO/IZO anode having similar electrical and optical properties to those of a pure IZO layer, it shows a higher work function than pure IZO due to the effect of the $\mathrm{NiO}_{x}$ in the NIZO layer. Furthermore, the current density-voltage-luminance $(J-V-L)$ curve and the efficiency of the

z E-mail: hkkim@kumoh.ac.kr
OLED fabricated on the NIZO layer demonstrates that the NIZO layer more effectively enhances hole injection compared to the pure IZO layer due to the high work function of NIZO.

\section{Experimental}

Two tilted magnetron guns were used to uniformly cosputter the IZO (5 wt \% ZnO-doped $\mathrm{In}_{2} \mathrm{O}_{3}$ ) and Ni targets (99.99\% purity). Prior to the deposition of the NIZO layer, a $135 \mathrm{~nm}$ thick IZO film was sputtered on a $25 \times 25 \mathrm{~mm}$ glass substrate at optimized conditions. During the deposition of the IZO film, the sputtering conditions were maintained constant at a working pressure of $5 \mathrm{mTorr}, \mathrm{dc}$ power of $100 \mathrm{~W}$, Ar flow rate of $20 \mathrm{sccm}$, and a substrate-to-target distance of $100 \mathrm{~mm}$. After the deposition of the IZO film at room temperature, a $5 \mathrm{~nm}$ thick NIZO layer was subsequently deposited on the IZO layer using a $\mathrm{Ni}$ and IZO dual-target sputtering process. Using a constant dc power for the IZO target $(100 \mathrm{~W})$, Ni atoms were doped into the IZO layer as a function of $\mathrm{Ni}$ target dc power, which was tuned from 0 to $7 \mathrm{~W}$. During the Ni and IZO cosputtering process, the Ar flow rate and working pressure were maintained constant at $20 \mathrm{sccm}$ and 5 mTorr, respectively. For simplicity, the IZO layer that was cosputtered at a $\mathrm{Ni}$ dc power of $3 \mathrm{~W}$ is referred to as " 3 W NIZO" and so on. The film thicknesses of the IZO and NIZO layers were obtained by using a stylus profilometer. The sheet resistance and resistivity of the NIZO/IZO anode were measured by a four-point probe and Hall measurement system using van der Pauw geometry at room temperature, respectively. The optical transmittance of the NIZO/IZO anode was measured in the wavelength range of $250-800 \mathrm{~nm}$ by a UV/visible spectrometer as a function of $\mathrm{Ni}$ dc power. The surface morphology of the NIZO layer was also characterized using a field-emission scanning electron microscope (FESEM) as a function of the $\mathrm{Ni}$ dc power. X-ray diffraction (XRD) and glancing angle XRD (GXRD) examinations were used to investigate the structural properties of the NIZO/IZO anode. The existence of $\mathrm{NiO}_{x}$ in the NIZO layer and the change in binding energy for the elements in the NIZO layer were examined by X-ray photoelectron spectroscopy (XPS-PHI5200) using an Al K $\alpha$ radiation source in an ultrahigh vacuum system with a base pressure of $\sim 10^{-10}$ Torr. The work function of the NIZO layer was measured using photoelectron spectroscopy with a UV source (PKI model $\mathrm{AC}-2)$ at atmospheric pressure. Conventional OLEDs were prepared on the NIZO layer and on a pure IZO anode (reference) to investi- 


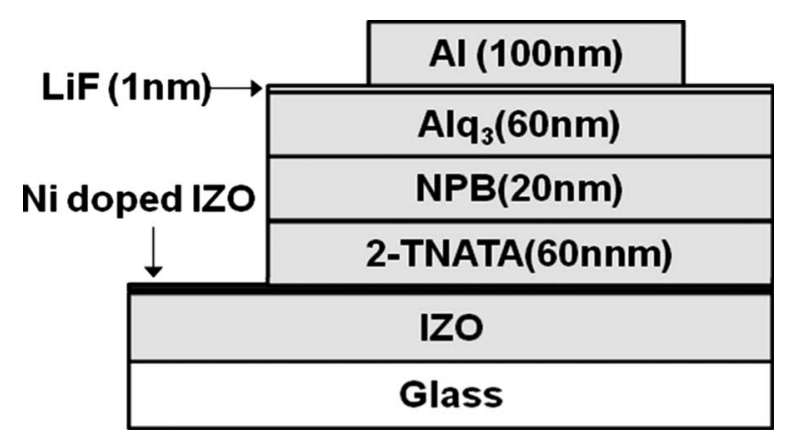

Figure 1. Schematic structure of the OLED fabricated on the Ni-doped IZO layer.

gate the impact of Ni doping on OLED characteristics. Figure 1 depicts the schematic multilayer structure of the OLED fabricated on the NIZO anode. After conventional wet cleaning and UV-ozone treatment, the reference IZO and NIZO/IZO layers were transferred to an organic/metal evaporation system. A $60 \mathrm{~nm}$ thick $4,4^{\prime}, 4^{\prime \prime}$-tris $[N$-(2-naphthyl)- $N$-phenyl-amino $]$-triphenylamine TNATA) film and $20 \mathrm{~nm}$ thick $\alpha$-naphthylphenlylbiphenyl film were grown on the reference IZO and NIZO layers as a hole injection layer and hole transport layer (HTL), respectively. Subsequently, a $60 \mathrm{~nm}$ thick tris-(8-hydroxyquinoline) aluminum $\left(\mathrm{Alq}_{3}\right)$ film and $1 \mathrm{~nm}$ thick LiF layer were grown on the HTL layer as emission layer and electron transporting layer (ETL). Finally, a $100 \mathrm{~nm}$ thick Al cathode layer was patterned using a shadow metal mask. The $J-V-L$ characteristics of the as-fabricated OLEDs were examined using a Photo Research PR-650 spectrophotometer driven by a programmable de source.

\section{Results and Discussion}

Figure 2 shows the sheet resistances and resistivities of the $5 \mathrm{~nm}$ thick NIZO layer grown on the $135 \mathrm{~nm}$ thick IZO anode at a constant IZO dc power of $100 \mathrm{~W}$, working pressure of $5 \mathrm{mTorr}$, and $\mathrm{Ar}$ flow rate of $20 \mathrm{sccm}$ as a function of $\mathrm{Ni}$ dc power. From this figure it can be observed that the sheet resistance and resistivity of $1 \mathrm{~W}$ $\mathrm{NIZO/IZO}$ is very similar to those of $0 \mathrm{~W}$ NIZO (pure IZO) due to the very low $\mathrm{Ni}$ sputtering rate at a $\mathrm{Ni}$ dc power of $1 \mathrm{~W}$. The $3 \mathrm{~W}$ NIZO layer exhibits a decreased sheet resistance and resistivity due to effective incorporation of $\mathrm{Ni}$ atoms into the IZO layer; however, $\mathrm{Ni}$ dc powers greater than $5 \mathrm{~W}$ lead to films with increased sheet resistance and resistivity. At $7 \mathrm{~W}$ of $\mathrm{Ni}$ dc power, abrupt increase in sheet resistance and resistivity are observed due to the formation of a large amount of the insulating $\mathrm{NiO}_{x}$ phase in the IZO matrix.

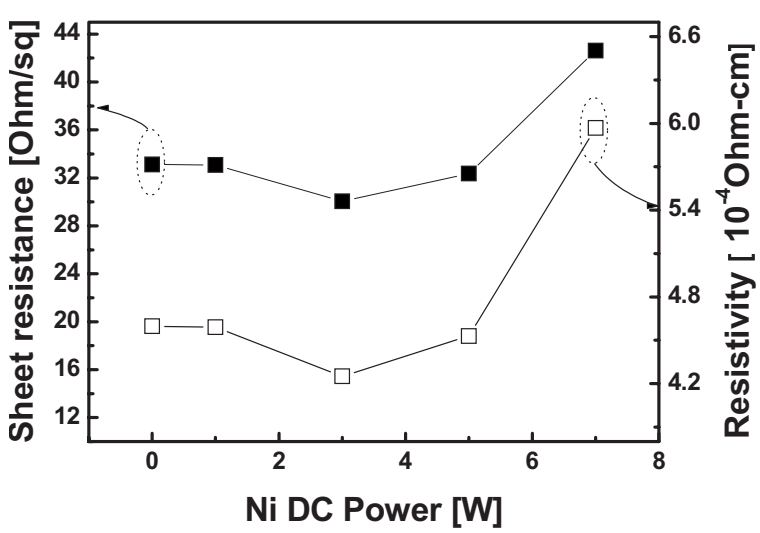

Figure 2. Sheet resistance and resistivity of the NIZO layer grown on the IZO anode as a function of $\mathrm{Ni}$ dc power from 0 to $7 \mathrm{~W}$ at a constant IZO dc power of $100 \mathrm{~W}$, working pressure of $5 \mathrm{mTorr}$, and Ar flow rate of $20 \mathrm{sccm}$.

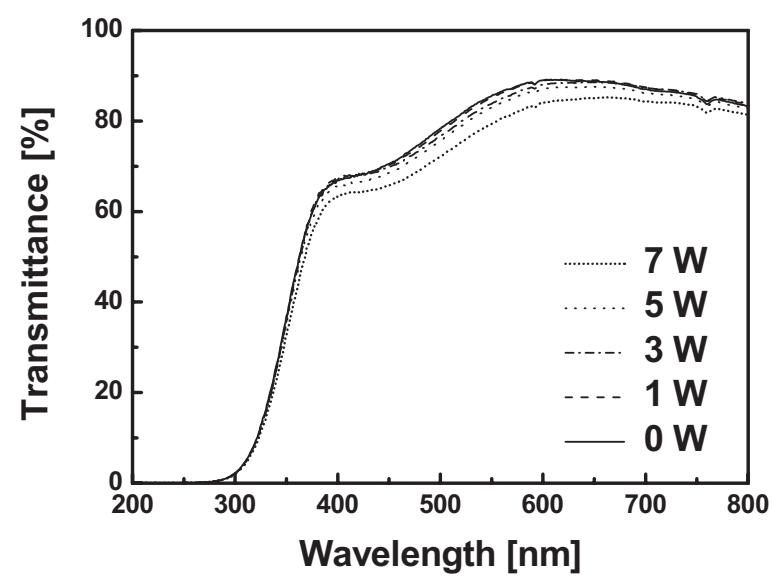

Figure 3. Optical transmittance of the NIZO/IZO multilayer anode as a function of $\mathrm{Ni}$ dc power.

Figure 3 depicts the optical transmittance spectra for the NIZO/ IZO anode grown at a constant dc power of $100 \mathrm{~W}$, working pressure of $5 \mathrm{mTorr}$, and Ar flow rate of $20 \mathrm{sccm}$ as a function of $\mathrm{Ni} \mathrm{dc}$ power. All NIZO/IZO anode films, except $7 \mathrm{~W}$ NIZO, exhibit comparable optical transmittance to pure IZO layer, regardless of the $\mathrm{Ni}$ dc power. At a wavelength of $550 \mathrm{~nm}$, the transmittance of the $\mathrm{NIZO/IZO} \mathrm{anode} \mathrm{grown} \mathrm{at} \mathrm{Ni} \mathrm{dc} \mathrm{powers} \mathrm{of} 1-3 \mathrm{~W}$ is $83.8 \%$. This observation indicates that the existence of the NIZO layer on the IZO had no affect on the optical properties of IZO anode layer; however, the IZO anode covered with a $7 \mathrm{~W}$ NIZO layer exhibits a decrease in optical transparency due to the formation of a relatively opaque $\mathrm{NiO}_{x}$ phase in the IZO layer. The sharp absorption edges in the transmittance spectra are caused by an extrinsic bandgap of the IZO layer in the range of 3.8-4.0 eV.

Both the XRD and glancing GXRD examinations were simultaneously employed to investigate the structure of the NIZO layer grown on the IZO anode. Figure 4a depicts the XRD plots obtained from the NIZO/IZO samples as a function of dc Ni power $(0-7 \mathrm{~W})$. All XRD plots of the NIZO/IZO anode exhibit two broad peaks, which are identical to spectra observed for the glass substrate and IZO layer. The broad peaks at $\sim 23.6$ and $31.7^{\circ}$ correspond to the amorphous glass and amorphous IZO covered with the NIZO layer. ${ }^{17}$ Due to the low substrate temperature during the Ni and IZO cosputtering process, all NIZO/IZO samples exhibit an amorphous structure, regardless of $\mathrm{Ni}$ dc power. The microstructure of the NIZO layer was also examined by GXRD analysis, as shown in Fig. $4 \mathrm{~b}$. As expected from the XRD results, the GXRD spectra are devoid of any peaks that allude to crystalline NIZO due to the low substrate temperature during the $\mathrm{Ni}$ and IZO cosputtering process. The surface smoothness of the NIZO layer grown on the IZO anode was analyzed using FESEM examination as a function of $\mathrm{Ni} \mathrm{dc}$ power (not shown here). The smooth surface of the anode layer in OLEDs is very important because the current injection stability and leakage current are critically influenced by surface defects such as protrusions or pinholes. In the case of the NIZO layer, all samples show an amorphous-like surface, which corroborates the aforementioned XRD results due to the low substrate temperature. In general, the pure IZO anode grown at room temperature showed a smooth and featureless surface due to the stable amorphous properties of the IZO film. ${ }^{17}$ The $1 \mathrm{~W}$ NIZO layer exhibited a similar surface image to the pure IZO layer, which indicates the $\mathrm{Ni}$ atom was not effectively doped into the IZO layer during the $\mathrm{Ni}$ and IZO cosputtering process due to a low $\mathrm{Ni}$ sputtering rate at $1 \mathrm{~W} \mathrm{Ni}$ dc power. However, the NIZO layer prepared at a Ni dc power greater than 3-7 W exhibits an agglomerated surface image, which was caused by $\mathrm{Ni}$ incorporation into the IZO layer.

Figure 5 depicts the XPS core-level spectra obtained from the surface of the NIZO layer grown on the IZO film as a function of dc 

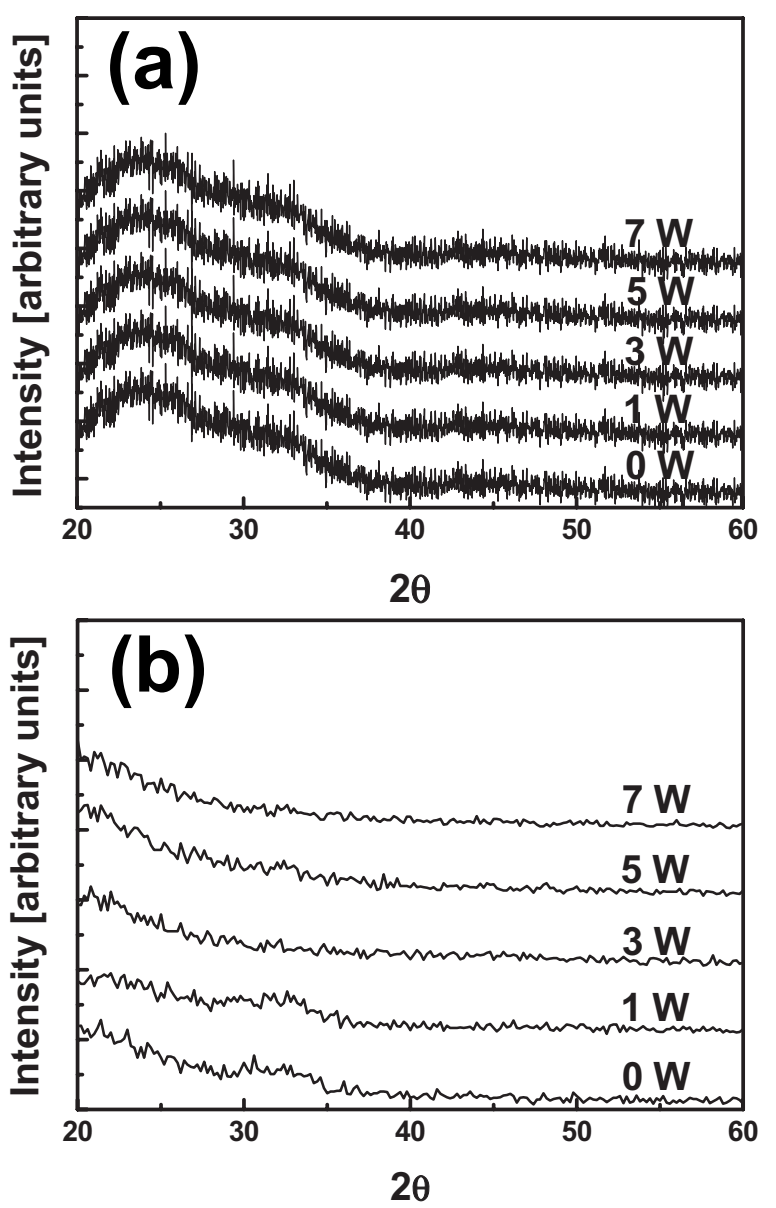

Figure 4. (a) XRD plots obtained from the NIZO/IZO multilayer anode grown at a constant IZO dc power of $100 \mathrm{~W}$, working pressure 5 mTorr, and Ar flow rate $20 \mathrm{sccm}$ as a function of $\mathrm{Ni}$ dc power. (b) GXRD plot using an incident angle of $5^{\circ}$ obtained from the surface region of the NIZO layer.
$\mathrm{Ni}$ power. A reference IZO (pure IZO anode) film was prepared for comparative purpose. The XPS core-level spectra data obtained from the NIZO layers shows In $3 \mathrm{~d}, \mathrm{O} 1 \mathrm{~s}, \mathrm{Zn} 2 \mathrm{p}$, and Ni 2p peaks. Compared to the In $3 \mathrm{~d}$ and $\mathrm{O} 1 \mathrm{~s}$ peaks, the peak intensities of the $\mathrm{Zn}$ $2 p$ and $\mathrm{Ni} 2 \mathrm{p}$ peaks are weak due to the low concentration of $\mathrm{Zn}$ and $\mathrm{Ni}$ in the NIZO layer. In the case of In $3 \mathrm{~d}$ and $\mathrm{Zn} 2 \mathrm{p}$ peaks, there are no changes in peak intensity, which indicate that In and $\mathrm{Zn}$ bindings are not affected by Ni cosputtering. In contrast, the $\mathrm{O} 1 \mathrm{~s}$ and $\mathrm{Ni} 2 \mathrm{p}$ peak intensities are affected by the dc power of the Ni atom during cosputtering, as shown in Fig. $5 \mathrm{c}$ and d. The two binding energies of 529.5 and $531.2 \mathrm{eV}$ in the $\mathrm{O} 1 \mathrm{~s}$ core-level spectra are caused by the two types of $\mathrm{O}^{2-}$ ions in the NIZO anode layer. It has been reported that the lower binding energy peak $\left(\mathrm{O}_{\mathrm{II}}\right)$ is from the $\mathrm{O}^{2-}$ ions, which have neighboring indium atoms with their full complement, while the higher binding energy peak $\left(\mathrm{O}_{\mathrm{I}}\right)$ corresponds to oxygen-deficient regions. ${ }^{17,18}$ Figure $5 \mathrm{c}$ demonstrates that the $\mathrm{O}_{\mathrm{II}} / \mathrm{O}_{\mathrm{I}}$ peak ratios of NIZO grown at $\mathrm{Ni}$ dc powers between 0 and $5 \mathrm{~W}$ are similar due to stable oxygen vacancies, which is the dominant component in the conductivity. In contrast, it was observed that the relative $\mathrm{O}_{\text {II }}$ peak intensity in the NIZO layer grown at a $\mathrm{Ni}$ dc power of $7 \mathrm{~W}$ decreased due to the large amount of cosputtered $\mathrm{Ni}$ atoms occupying oxygen vacancy sites. ${ }^{14}$ In addition, we observed that the intensity of the Ni $2 p$ peak obtained from the NIZO layer increased as a function of increasing $\mathrm{Ni}$ dc power, as shown in Fig. 5d. The NIZO layer grown at a $\mathrm{Ni}$ dc power greater than $3 \mathrm{~W}$ exhibited a $\mathrm{Ni} 2 \mathrm{p}$ peak, and a satellite peak at an identical position appeared at 854.6 and $861.0 \mathrm{eV}$, respectively. Wang et al., investigating the structure of $\mathrm{Ni}$-doped $\mathrm{ZnO}$ nanocrystals, reported that the $\mathrm{Ni} 2 \mathrm{p}_{3 / 2}$ peak and satellite peak observed at $\sim 855.4$ and $\sim 861.5 \mathrm{eV}$ are related to the oxidized state of the $\mathrm{Ni}$ atom because the metallic $\mathrm{Ni} 2 \mathrm{p}_{3 / 2}$ peaks should appear at $852.7 \mathrm{eV} .{ }^{19}$ The peaks observed in our investigation indicate that the cosputtered $\mathrm{Ni}$ atoms were incorporated into the NIZO layer as a $\mathrm{NiO}_{x}$ phase; however, there is no $\mathrm{Ni} 2 \mathrm{p}$ peak and satellite peak related to the $\mathrm{NiO}_{x}$ phase in the $1 \mathrm{~W}$ NIZO layer because of poor Ni doping into the IZO layer due to the low Ni sputtering rate. Therefore, it is clear that a $\mathrm{Ni}$ dc power above $3 \mathrm{~W}$ is necessary for doping $\mathrm{Ni}$ atoms into an IZO layer during the cosputtering of Ni and IZO dual targets. Table I summarizes the elemental compositions of the NIZO layer as a function of $\mathrm{Ni} d c$
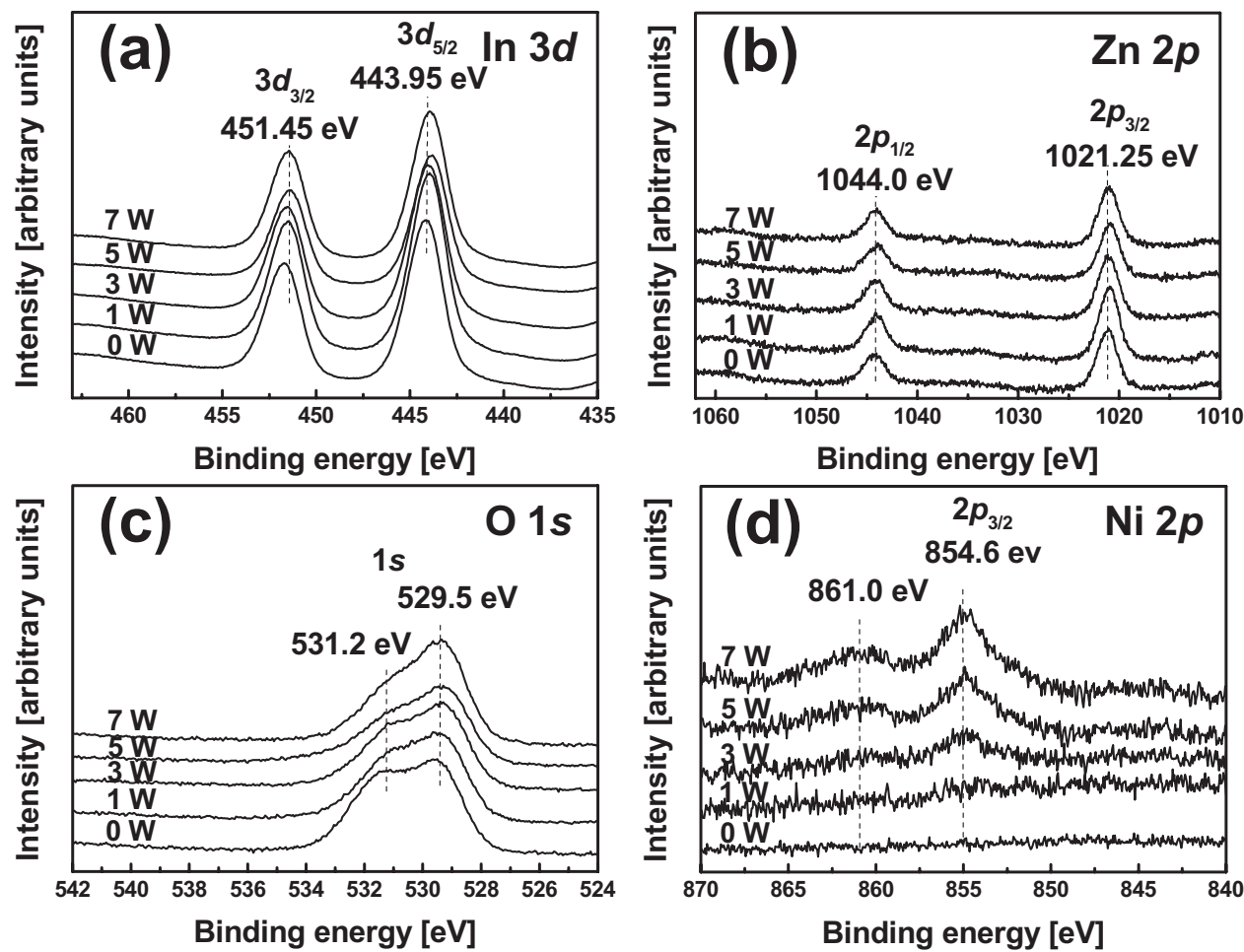

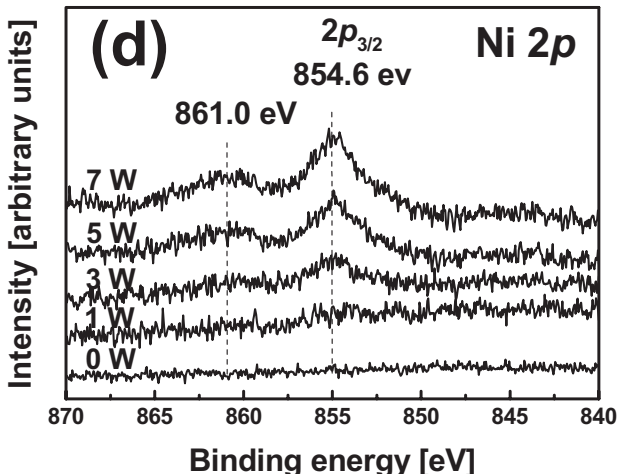

Figure 5. XPS spectra of (a) In 3d, (b) Zn $2 \mathrm{p}$, (c) $\mathrm{O} 1 \mathrm{~s}$, and (d) Ni $2 \mathrm{p}$ obtained from the surface region of the NIZO layer grown at a constant IZO dc power of $100 \mathrm{~W}$, working pressure 5 mTorr, and $\mathrm{Ar}$ flow rate $20 \mathrm{sccm}$ as a function of $\mathrm{Ni} \mathrm{dc}$ power. 


\begin{tabular}{|c|c|c|c|c|}
\hline \multirow{2}{*}{$\begin{array}{l}\text { Sample } \\
\text { (Ni power) }\end{array}$} & \multicolumn{4}{|c|}{ Atomic percentage $(\%)$} \\
\hline & In $3 d$ & $\mathrm{Zn} 2 \mathrm{p}$ & $\mathrm{O} 1 \mathrm{~s}$ & $\mathrm{Ni} 2 \mathrm{p}$ \\
\hline $0 \mathrm{~W}$ & 33.50 & 2.63 & 63.87 & 一 \\
\hline $1 \mathrm{~W}$ & 33.61 & 2.96 & 63.42 & - \\
\hline $3 \mathrm{~W}$ & 33.67 & 2.72 & 62.63 & 0.98 \\
\hline $5 \mathrm{~W}$ & 33.36 & 2.81 & 62.15 & 1.68 \\
\hline $7 \mathrm{~W}$ & 34.11 & 2.98 & 60.62 & 2.28 \\
\hline
\end{tabular}

power. As expected from the $\mathrm{Ni} 2 \mathrm{p}$ core-level spectra, the atomic percentage of $\mathrm{Ni}$ in the NIZO layer begins to appear at a $\mathrm{Ni} d c$ power of $3 \mathrm{~W}$.

Figure 6 depicts the work function of the NIZO layer as a function of $\mathrm{Ni}$ de power. The work functions of the 0 and $1 \mathrm{~W}$ NIZO layers are 4.98 and $5.04 \mathrm{eV}$, respectively, which are higher than that of conventional ITO $(4.5-4.8 \mathrm{eV})$. The 3 and $5 \mathrm{~W}$ NIZO layers exhibited increased work function to 5.15 and $5.18 \mathrm{eV}$, respectively. An increase of $\mathrm{Ni}$ dc power to $7 \mathrm{~W}$ increases the work function to $5.25 \mathrm{eV}$. It has been reported that the work function of an amorphous IZO layer is approximately $4.8-5.0 \mathrm{eV}$, while $\mathrm{Ni}$ is around $5.2 \mathrm{eV} .{ }^{17} \mathrm{Hsu}$ et al. reported that the work functions of Ni-doped ITO anode layers were significantly affected by Ni-doping concentration. ${ }^{14}$ Hence, the increased work function of the NIZO layer is most likely due to the effect of $\mathrm{Ni}$ doping into the IZO layer. In order to make high-performance OLEDs, it is necessary to employ a transparent conducting oxide anode layer with a high work function greater than $5 \mathrm{eV}$, because hole injection critically depends on the barrier height formed between the anode and the organic materials. Therefore, the NIZO layer with a higher work function than that of pure IZO enhances hole injection into OLEDs. Although the $7 \mathrm{~W}$ NIZO layer exhibits the highest work function, we decided that the $3 \mathrm{~W}$ NIZO layer represents the optimized cosputtered anode because it has a much lower sheet resistance and higher transmittance than those of the 5 and $7 \mathrm{~W}$ NIZO layers.

Conventional OLEDs were fabricated onto the NIZO layer and IZO (reference) anode to investigate a NIZO/IZO layer as an anode in OLEDs. Figure 7 a depicts the $J-V$ characteristics of the OLEDs fabricated on the $3 \mathrm{~W}$ NIZO and reference IZO anodes, respectively. The $J-V$ curves of the OLEDs fabricated on the $3 \mathrm{~W}$ NIZO layer shows a lower turn-on voltage and a higher current density than

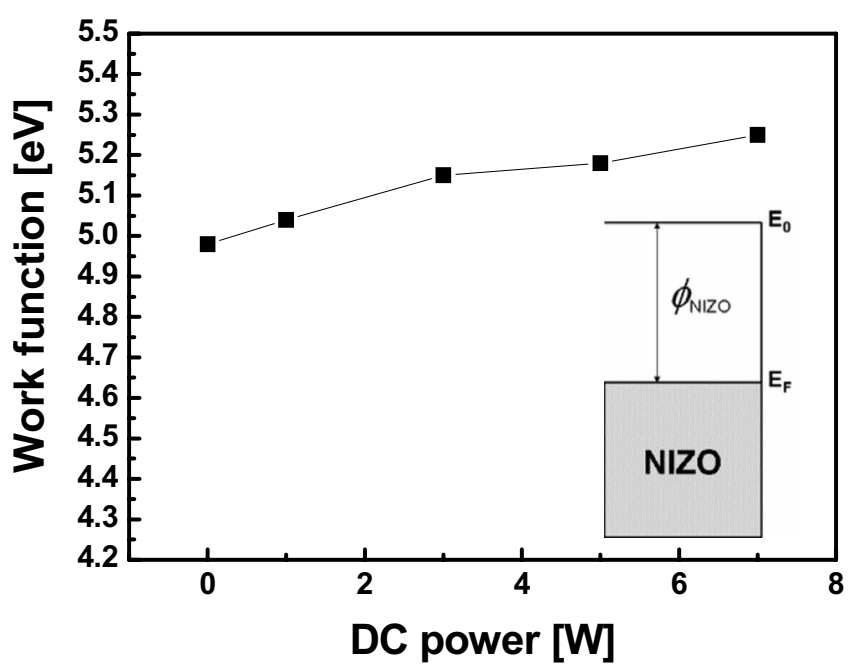

Figure 6. Work function of the NIZO layer grown at a constant IZO dc power of $100 \mathrm{~W}$, working pressure $5 \mathrm{mTorr}$, and Ar flow rate $20 \mathrm{sccm}$ as a function of $\mathrm{Ni}$ de power.
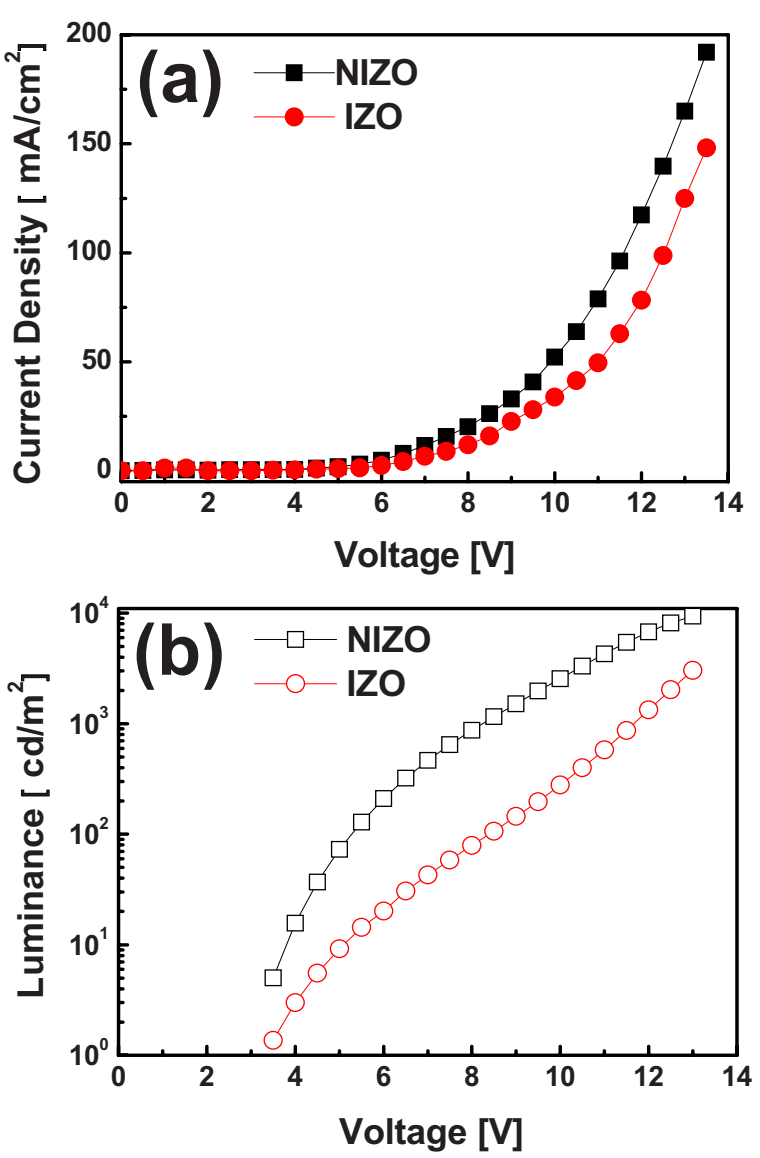

Figure 7. (Color online) (a) $J-V$ and (b) $L-V$ curves of an OLED fabricated on a $3 \mathrm{~W}$ NIZO layer (optimized condition) and reference IZO anode.

those of the OLED fabricated on reference IZO anode. These observations indicate that hole injection from the anode is enhanced by introducing the NIZO layer with a higher work function between the 2-TNATA and the IZO anode. The luminance-voltage $(L-V)$ curve depicted in Fig. 7b also demonstrates that the luminance of the OLED fabricated on the NIZO layer is higher than that of the OLED fabricated on the IZO anode, as expected from the $J$ - $V$ curve. In addition, the OLED prepared on $3 \mathrm{~W}$ NIZO anode showed a higher external quantum efficiency (EQE) of $1.83 \%$ than that of the reference OLED $(1.03 \%)$ due to the higher hole injection efficiency of the NIZO layer. The emission spectra (not shown here) of the green OLED fabricated on the NIZO layer is identical to that obtained from the reference sample. Herein, there is no observable shift in the emission spectra of the OLED fabricated on the NIZO layer, which indicates that the optical characteristics of the green OLED are not affected by the NIZO layer introduced between the 2TNATA and IZO anode. This is also confirmed by the identical CIE coordinates of OLEDs fabricated on the NIZO layer and IZO anode.

The improved $J-V-L$ characteristics and EQEs of OLED fabricated on the NIZO layer are related to the higher work function of the NIZO layer compared to that of the amorphous IZO anode. The low barrier height between the NIZO layer and the 2-TNATA potentially enhances hole injection into the organic layer. In addition, the formation of a p-type $\mathrm{NiO}_{x}$ phase in the NIZO layer could be responsible for enhancing hole injection into the OLEDs. Chan et al., suggested that passibility of the $\mathrm{NiO}_{x}$ layer formation in the ITO anode could enhance the hole injection in OLEDs because $\mathrm{NiO}_{x}$ is a p-type transparent conducting oxide with a high work function. ${ }^{15}$ In comparison to an n-type ITO or IZO layer, the p-type $\mathrm{NiO}_{x}$ could inject hole carriers more easily into the organic layer. Formation of the $\mathrm{NiO}_{x}$ phase in the NIZO layer, which was confirmed by the 
existence of the Ni $2 p$ and satellite peaks shown in Fig. 5d, is responsible for the enhancement of hole injection into the organic layer. Therefore, the combined effect of a high-work-function NIZO layer and uniformly distributed $\mathrm{NiO}_{x}$ phase in the NIZO enabled us to fabricate high-performance OLEDs.

\section{Conclusion}

This report discussed the characteristics of NIZO layers and demonstrated the applicability of a NIZO/IZO multilayer structure as an anode for high-performance OLEDs. Although the Ni and IZO were cosputtered at room temperature, we obtained an NIZO/IZO anode with a sheet resistance of $30.04 \Omega / \square$ and an average optical transmittance of $83.8 \%$ at a wavelength of $550 \mathrm{~nm}$. In addition, it was observed that the existence of a NIZO layer on the IZO anode did not impact the electrical and optical properties of the IZO layer at low $\mathrm{Ni}$ cosputtering powers of $1-5 \mathrm{~W}$. The introduction of a NIZO layer between the organic and IZO anode layer could potentially enhance hole injection into OLEDs via the high work function of the NIZO and formation of a p-type $\mathrm{NiO}_{x}$ phase in the NIZO layer.

\section{Acknowledgment}

The authors acknowledge financial support from LG Displays, OLED Panel Development team.
Kumoh National Institute of Technology assisted in meeting the publication costs of this article.

\section{References}

1. C. W. Tang and S. A. VanSlyke, Appl. Phys. Lett., 51, 913 (1987).

2. H.-K. Kim and K.-S. Lee, Electrochem. Solid-State Lett., 11, J57 (2008).

3. H. Kim, C. M. Gilmore, A. Pique, J. S. Horwitz, H. Mattoussi, H. Murata, Z Kafafi, and D. B. Chrisey, J. Appl. Phys., 86, 6451 (1999).

4. R. B. H. Tahar, T. Ban, Y. Ohya, and Y. Takahashi, J. Appl. Phys., 83, 2631 (1998).

5. H. Kim, J. S. Horwitz, G. P. Kushto, Z. H. Kafafi, and D. B. Chrisey, Appl. Phys. Lett., 79, 284 (2001)

6. H. Y. Yu, X. D. Feng, D. Grozea, Z. H. Lu, R. N. S. Sodhi, A.-M. Hor, and H. Aziz, Appl. Phys. Lett., 78, 2595 (2001).

7. F. Li, H. Tang, J. Shinar, O. Resto, and S. Z. Weisz, Appl. Phys. Lett., 70, 2741 (1997).

8. C. Ganzorig, K.-J. Kwak, K. Yagi, and M. Fujihira, Appl. Phys. Lett., 79, 272 (2001).

9. I.-M. Chan, W.-C. Cheng, and F. C. Hong, Appl. Phys. Lett., 80, 13 (2002).

10. C. C. Wu, I. Wu, J. C. Sturn, and A. Kahn, Appl. Phys. Lett., 70, 1348 (1997).

11. J.-M. Moon, J.-H. Bae, J.-A. Jeong, S.-W. Jeong, N.-J. Park, H.-K. Kim, J.-W. Kang, J.-J. Kim, and M.-S. Yi, Appl. Phys. Lett., 90, 163516 (2007).

12. S. F. J. Appleyard and M. R. Willis, Opt. Mater, 9, 120 (1998).

13. C.-M. Hsu and W.-T. Wu, Appl. Phys. Lett., 85, 840 (2004).

14. C.-M. Hsu, J.-W. Lee, T.-H. Meen, and W.-T. Wu, Thin Solid Films, 474, 19 (2005).

15. I.-M. Chan, T.-Y. Hsu, and F. C. Hong, Appl. Phys. Lett., 81, 1899 (2002).

16. H. Sato, T. Minami, S. Takata, and T. Yamada, Thin Solid Films, 236, 27 (1993).

17. J.-H. Bae, J-M. Moon, J-W. Kang, H.-D. Park, J.-J. Kim, W. J. Cho, and H.-K. Kim, J. Electrochem. Soc., 154, J81 (2007).

18. J. C. C. Fan and J. B. Goodenough, J. Appl. Phys, 48, 3524 (1977).

19. H. Wang, Y. Chen, H. B. Wang, C. Zhang, F. J. Yang, J. X. Duan, C. P. Yang, Y. M. Xu, M. J. Zhou, and Q. Li, Appl. Phys. Lett., 90, 052505 (2007). 